\title{
Effects of Azolla spp. as feed ingredient on the growth performance and nutrient digestibility of broiler chicken
}

\begin{abstract}
The feeding trial was conducted to investigate the effect of Azolla spp. inclusion in feed ingredient of broiler chickens. Two hundred of day-old broiler chicks were raised in Farm 15, Faculty of Agriculture, Universiti Putra Malaysia. The birds were individually wing banded, weighed and allocated into four treatment groups (control: 0\% of Azolla spp., T1: 5\% of Azolla spp., T2: 10\% of Azolla spp. and T3: 15\% of Azolla spp.). Each group has five replicates and ten birds per pen. All the birds were fed ad libitum during the feeding trial, which lasted 42 days. During the end of the starter and finisher phase, two birds from each replicate were slaughtered. Ileal digesta sample was collected to determine the nutrient digestibility of the Azolla spp. on broiler chickens. Azolla spp. nutritional content was $5.14 \%$ dry matter, $24.82 \%$ crude protein, $16.64 \%$ crude fibre, $2.00 \%$ ether extract, $11.59 \%$ ash, $34.95 \%$ nitrogen-free extract, $42.52 \%$ neutral detergent fibre, $31.15 \%$ acid detergent fibre, $16.86 \%$ acid detergent lignin, $11.37 \%$ hemicellulose and $14.29 \%$ cellulose. The result from this study showed T3 (15\% of Azolla spp.) has significantly highest $(\mathrm{p}<.05)$ in overall body weight gain. However, in terms of feed intake and feed conversion ratio, there were no significant differences $(\mathrm{p}>.05)$ among treatments. For the nutrient digestibility experiment, the findings showed compared with a control group, feeding of $10 \%$ or $15 \%$ of Azolla spp. led to significant improvement $(\mathrm{p}<.05)$ in nutrient digestibility. The studies conclude the inclusion of Azolla spp. up to $15 \%$ in broiler chicken feed ration can improved growth of body performance and showed no adverse effect on the growth performance and nutrient digestibility of the birds.
\end{abstract}

Keyword: Azolla spp.; Body weight gain; Broiler chicken; Growth performance; Nutrient digestibility 